\title{
Inter and Intra Cluster Scheduling for Performance Analysis of Coexistence Heterogeneous Networks
}

\author{
G.M.Tamilselvan \\ Lecturer, Department of ECE \\ Bannariamman Institute of Technology \\ Sathyamangalam, India
}

\author{
Dr.A.Shanmugam \\ Principal \\ Bannariamman Institute of Technology \\ Sathyamangalam, India
}

\begin{abstract}
In ubiquitous networking environments, we generally need two or more heterogeneous communication systems coexisting in a single place. Especially, wireless local area networks (WLANs) based on IEEE $802.11 \mathrm{~b}$ specifications and wireless personal area networks (WPANs) based on IEEE 802.15.4 specifications need to coexist in the same Industrial, Science and Medial (ISM) band. If the WPAN communication coverage is expanded using a cluster-tree network topology, then the 802.15.4 network is more susceptible to interference from neighboring WLANs. In this paper, we propose an adaptive transmission power aware cluster scheduling algorithm using multiple channels in a WPAN in the presence of WLAN interference. The algorithm includes node identification, channel allocation, clustering and time scheduling. To evaluate the performance of the proposed algorithm, the performance metrics such as Bit error, Throughput, Average EndEnd Delay and Average Jitter is measured through Qualnet simulation. The measurement result shows that the proposed algorithm is effective in an IEEE 802.15.4 cluster-tree network in the presence of multiple IEEE 802.11 interferers.
\end{abstract}

Key Words

Clustering, Coexistence, Heterogeneous, WLAN and WPAN (Zigbee).

\section{INTRODUCTION}

As a low-power and low-cost technology, IEEE 802.15.4 is establishing its place on the market as an enabler for the emerging wireless sensor networks (WSNs) [1]. Like IEEE 802.11b and IEEE 802.11g, IEEE 802.15.4 is also used in the 2.4 $\mathrm{GHz}$ ISM band. Due to supporting complimentary applications, they are very likely to be collocated within the interfering range of each other and therefore their ability to coexist needs to be evaluated. In this paper we focus on the coexistence between these two major wireless standards that operate in the $2.4 \mathrm{GHz}$ ISM band. Their overlapping frequency channels are shown in Figure.1.

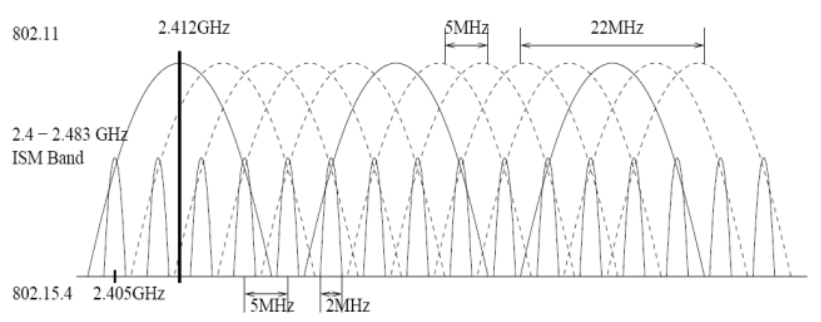

Figure.1.802.11b and 802.15.4 channels in the 2.4GHz ISM band.

There have been some studies about coexistence between the IEEE 802.11b and IEEE 802.15.4. According to [1] [2] [4] IEEE 802.15.4 has a little impact on the IEEE 802.11 performance. However, IEEE 802.11 can have a serious impact on the IEEE 802.15.4 performance if the channel allocation is not carefully taken into account [1] [3]. While the conclusion is true in general, we believe the studies so far have dealt with only limited cases of coexistence scenarios. In [3], the Packet Error Rate (PER) of IEEE 802.15.4 under the IEEE 802.11b interference is analyzed from an assumption of blind transmissions, i.e. both IEEE 802.11b and IEEE 802.15.4 transmit packets regardless of whether the channel state is busy or not. In [4], measurements are performed to quantify coexistence issues. Channel Conflict Probabilities between IEEE 802.15 based Wireless Personal Area Networks is modeled in [5]. Packet Error Rate of IEEE 802.15.4 under IEEE 802.11b interference is analyzed in [6].In [7] Packet Error Rate of IEEE 802.11b under IEEE 802.15.4 interference is analyzed. In [8] channel conflict probabilities between IEEE 802.11b and IEEE 802.15.4 have been modeled. In [9] channel collision between IEEE 802.15.4 and IEEE 802.11b for circular and grid topology is analyzed with the mobility model. The effect of inter packet delay is analyzed in [10].

The author concluded that despite its low transmit power and simple modulation technique, IEEE 802.15.4 shows a robust behavior against interference of other $2.4 \mathrm{GHz}$ systems and even in the worst case conditions for frequency overlap, local distance and high traffic load for interference, some time slots remain for a successful transmission of IEEE 802.15.4.In above said related works only two WPAN nodes which are collocated with multiple WLAN nodes are considered. But today the sensor networks play a vital role in any automation; we have to consider the multiple WPAN nodes. When multiple sensor nodes are used, time slot 
mechanism is not helpful in WPAN network because ZigBee is a mesh networking technology.

The remainder of the paper is organized as follows: Section 2 gives an overview of the IEEE 802.11b and IEEE 802.15.4 standard. Section 3 presents a cluster tree network with two different time and frequency scheduling schemes. Simulation results are shown in Section 4. Our conclusion is drawn in Section 5.

\section{OVERVIEW OF IEEE 802.11b AND IEEE 802.15.4}

\subsection{IEEE $802.11 b$}

IEEE 802.11b standard defines the Medium Access Control (MAC) sub layer and the Physical (PHY) layer for wireless LANs. The standard operates at 13 overlapping channels in the $2.4 \mathrm{GHz}$ ISM band and the bandwidth of each channel is 22 MHz. IEEE 802.11b MAC employs the Carrier Sense Multiple Access with Collision Avoidance (CSMA/CA) mechanism. Before initiating a transmission, an IEEE 802.11b node senses the channel to determine whether another node is transmitting. If the medium is sensed idle for a Distributed coordination function Inter-Frame Space (DIFS) time interval the transmission will be preceded. If the medium is busy the node defers its transmission. When the medium becomes idle for a DIFS interval, the node will generate a random back off delay uniformly chosen in an interval. This interval $[0, \mathrm{~W}]$ is called Contention Window, where $\mathrm{W}$ is the size of the contention window. The initial $\mathrm{W}$ is set to CWmin. The back off timer is decreased by one as long as the medium is sensed idle for a back off time slot. The back off counter will become frozen when a transmission is detected on the medium, and resumed when the channel is sensed idle again for a DIFS interval. When the back off timer reaches zero, the node transmits a DATA packet. Immediately after receiving a packet correctly, the destination node waits for a Short Inter Frame Spacing (SIFS) interval and then transmits an ACK back to the source node.

\subsection{IEEE 802.15.4}

IEEE 802.15.4 standard defines the MAC sub layer and the PHY layer for low-rate wireless personal area networks. Its operational frequency band includes the $2.4 \mathrm{GHz}$ ISM band. Like IEEE 802.11b, IEEE 802.15.4 also employs CSMA/CA for media access control. However there is a key difference between their CSMA/CA mechanisms. Unlike in IEEE 802.11b, a channel in IEEE 802.15.4 is not sensed during a back off period but only during a Clear Channel Assessment (CCA) period. Furthermore, the contention window in IEEE 802.15.4 is doubled correspondingly whenever the channel is determined busy during a CCA period. In IEEE 802.11b, however, the contention window remains the same size when the channel is determined busy and is doubled only when ACK is not received. This difference has a significant impact on their behavior of sharing a channel, which we shall show in detail at the following sections.

\section{PROPOSED SCHEME}

In this paper, we propose power aware time slot and frequency based spectrum access analysis for the performance metrics such as bit error, throughput, average End-End delay and average jitter of IEEE 802.15.4. In this proposed scheme the WPAN devices are clustered. Each cluster will have one PAN coordinator and four end devices. We consider a heterogeneous network with random topology.IEEE 802.15.4 topologies are shown in Figure.2 Here the performance of IEEE 802.15.4 under the interference of IEEE $802.11 \mathrm{~b}$ and the interference among IEEE 802.15.4 nodes because of multiple transmissions is analyzed using Qualnet 4.5 simulation. For simulation, the slotted CSMA/CA of the IEEE 802.15.4 model is developed using Qualnet 4.5.The random topology scenario of coexistence heterogeneous network with 20 WPAN and 20 WLAN nodes for two different scheduling schemes are shown in Figure. 4 \&5.
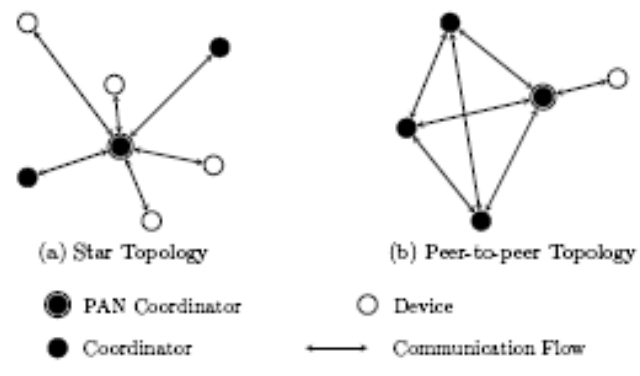

Figure.2. IEEE 802.15.4 Topologies

The PHY of the IEEE 802.15.4 at 2.4 GHz uses offset quadrature phase shift keying (OQPSK) modulation. Denote that the $E_{b} / N_{0}$ is the ratio of the average energy per information bit to the noise power spectral density at the receiver input, in the case of an additive white Gaussian noise (AWGN) channel. Then the bit error rate (BER), $P_{B}$, can be expressed as

$$
P_{B}=Q\left(\sqrt{\frac{2 E_{b}}{N_{\mathrm{O}}}}\right)
$$

Where $\mathrm{Q}(\mathrm{x})$ is

$Q(x)=\frac{1}{\sqrt{x}} \int_{x}^{\infty} \exp \left(\frac{-u^{2}}{2}\right) d u$

Figure. 3 shows the relationship between the bit error rate and $\mathrm{E}_{\mathrm{b}} / N_{o}$ simulated in Matlab. The bit error rate decreases when $E_{b}$ $/ N_{o}$ increases. The noise power spectral density increases when collision increases. As the number of WLAN sources increases, the BER of IEEE 802.15.4 increases because contentions among multiple WLANs increase the channel usage and cause collisions, which is more powerful interference, source to. 


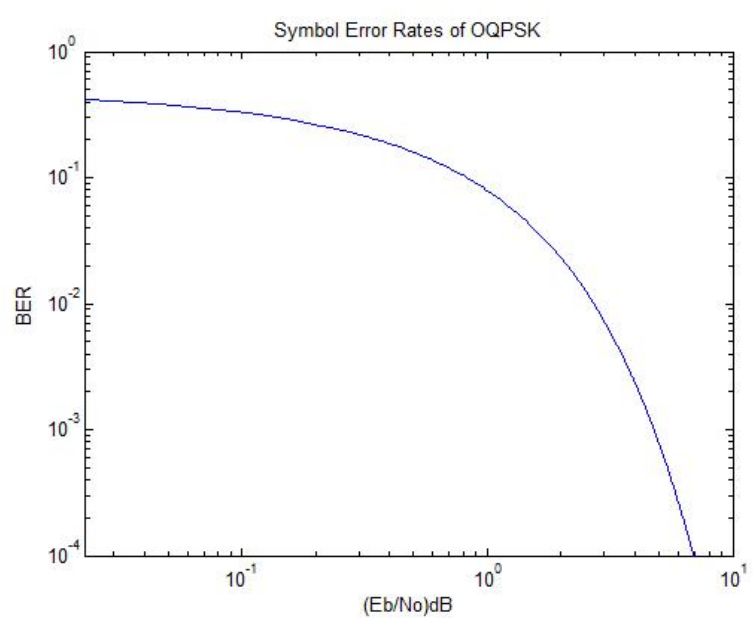

Figure.3. Theoretic Bit Error Rate of OQPSK

In this paper two schemes are proposed and the results are compared. The first scheme is called inter cluster scheduling. In this scheme the nodes are separated based on their transmission power. The output power of 802.15 .4 devices is typically as low as $0 \mathrm{dBm}$, whereas the output power of $802.11 \mathrm{~b}$ devices is 15 $d B m$ or above. Then WLAN nodes are grouped under one operating frequency and WPAN nodes are clustered with cluster size 5.Each cluster will have one PAN coordinator and four end devices. Each cluster is allotted unique channel frequency for error free transmission. After frequency scheduling, in each channel specific time slot is allotted for packet transmission.

In second scheme, intra cluster scheduling is considered. In this scheduling scheme the cluster members from different clusters are grouped under one channel and specific time slots are allotted for packet transmission. The figure $4 \& 5$ shows the scenario for two different schemes.

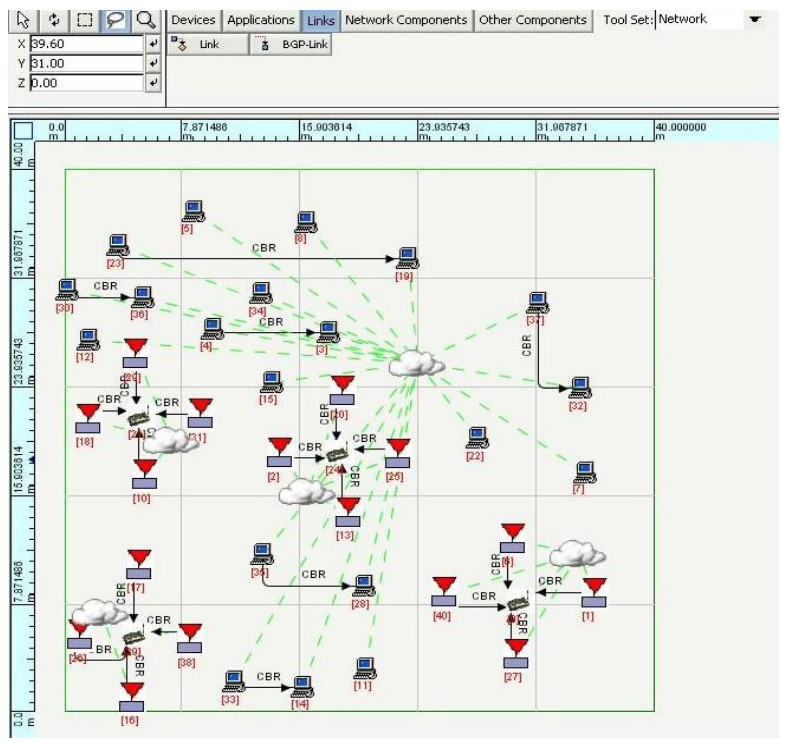

Figure.4.Random topology scenario with Inter cluster Scheduling

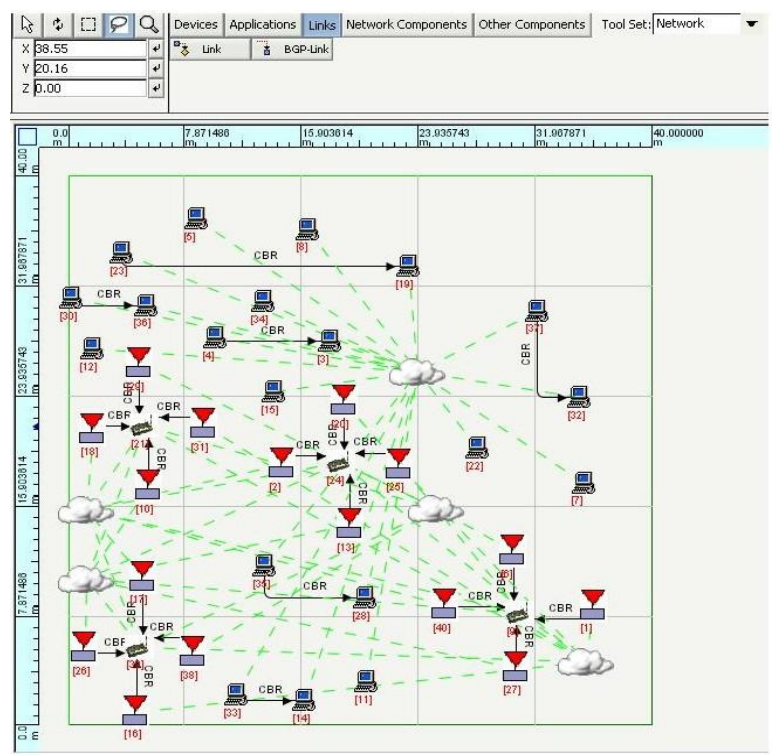

Figure.5.Random topology scenario with Intra cluster Scheduling

\section{SIMULATION RESULTS AND DISCUSSION}

To evaluate the effectiveness of the proposed scheme in a coexistence heterogeneous wireless network, a simulation study was conducted using Qualnet 4.5 simulator. The simulation configuration and parameters used in this paper is shown in Table 1.

Table 1.Simulation Configuration and Parameters

\begin{tabular}{|l|l|l|}
\hline \multicolumn{1}{|c|}{ Parameter } & IEEE 802.11b & IEEE 802.15.4 \\
\hline Number of Nodes & 20 & 20 \\
\hline Transmission Power & $15 \mathrm{dbm}$ & $3 \mathrm{dbm}$ \\
\hline Modulation & CCK & OQPSK \\
\hline MAC Protocol & 802.11 & 802.15 .4 \\
\hline Routing Protocol & Bellman ford & AODV \\
\hline No of Packets & 100 & 100 \\
\hline Payload Size & $1500 \mathrm{bytes}$ & $105 \mathrm{bytes}$ \\
\hline Simulation Time & $35 \mathrm{~s}$ & \\
\hline Packet Interval & $100 \mathrm{~ms}$ & $1 \mathrm{~ms}$ \\
\hline $\begin{array}{l}\text { Packet Transmission } \\
\text { Time }\end{array}$ & $5 \mathrm{~s}$ & $1 \mathrm{~s}$ \\
\hline Test bed size & $40 \mathrm{~m} \times 40 \mathrm{~m}$ & \\
\hline Topology & Random \\
\hline
\end{tabular}

The effectiveness of the proposed scheme was measured with four different metrics: Bit error, Throughput, Average End-End delay and Average jitter. The figure 6-9 shows the performance analysis of random topology with two different proposed schemes. 
The figure. 6 shows the bit error analysis for random topology. In this figure bit error for two different schemes namely inter cluster scheduling and intra cluster scheduling is shown. When the intra cluster scheduling is adopted the bit error becomes zero.

Bit Error in bits

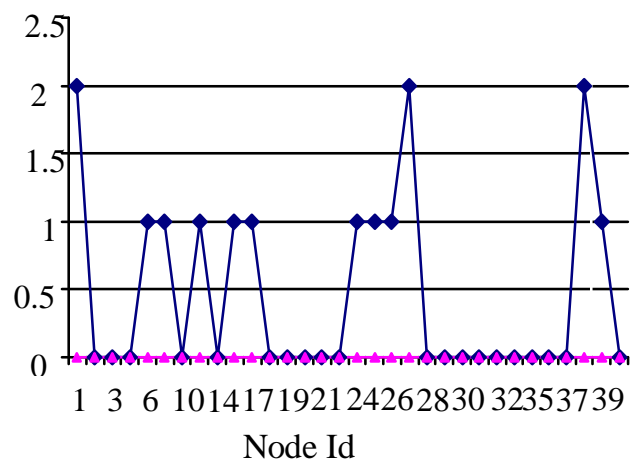

Inter cluster Scheduling $\rightarrow$ Intra Cluster Scheduling

Figure .6.Bit Error Analysis for Random Topology

When inter cluster scheduling is adopted time slot mechanism is not helpful in WPAN network because ZigBee is a mesh networking technology, which means that devices can automatically route messages on each other's behalf (often called multi-hopping). This allows deploying larger networks without immoderately increasing the transmission power since direct communications occur only in a geographically-restricted area.

Throughput in

bits/sec

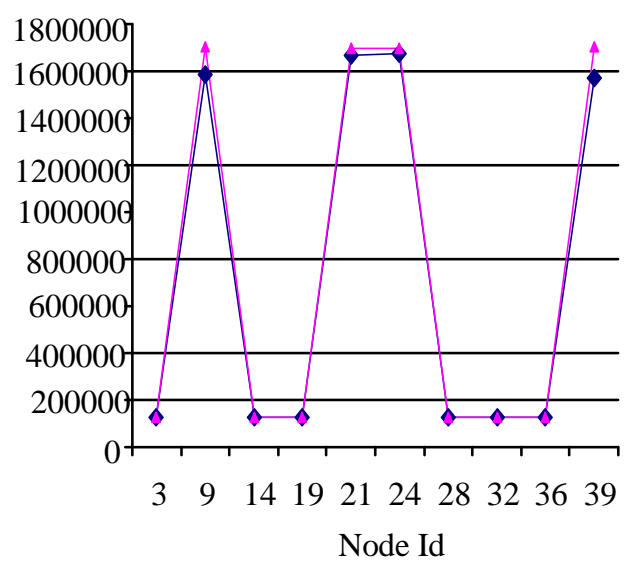

Inter cluster Scheduling $\rightarrow$ Intra Cluster Scheduling

\section{Figure .7.Throughput Analysis for Random Topology}

Throughput analysis for random topology with inter and intra cluster scheduling is shown in figure.7.The analysis shows that the throughput is considerably increasing in the case of intra cluster scheduling. After the implementation of this scheduling scheme throughput is increased by $7.34 \%$. The average end-end analysis is shown in figure. 8 .

Average End-End delay in sec

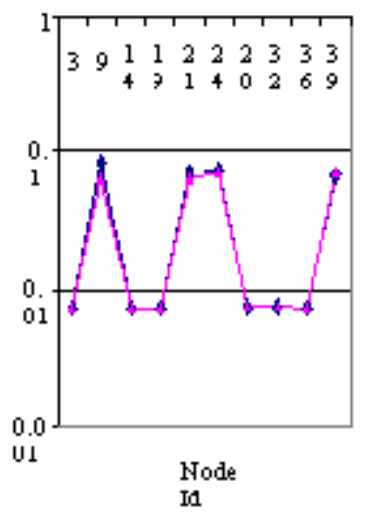

$\multimap$ Inter cluster Scheduling $\rightarrow$ Intra Cluster Scheduling

\section{Figure .8.Average End-End Delay Analysis for Random Topology}

The average end-end delay for WPAN and WLAN nodes are shown in figure. 8 . The average end-end delay is decreased by $5 \%$ $25 \%$ for WPAN nodes when intra clustering scheme is used. In figure.9.average jitter is given.

The figure. 9 show that the average jitter goes down by $4.7 \%-17.4$ $\%$.The intra clustering is working probably and the interference is completely mitigated.

Average Jitter in sec

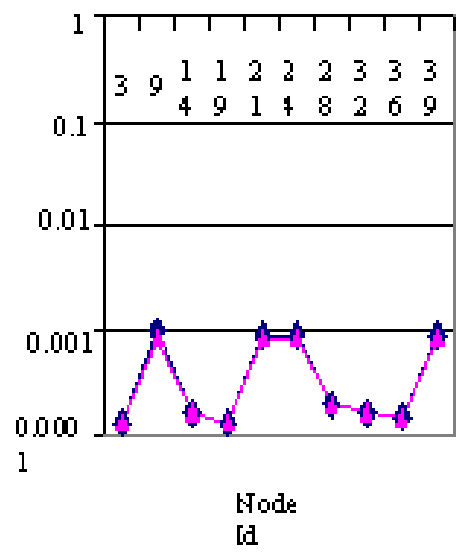

Inter cluster Scheduling $\_$Intra Cluster Scheduling

Figure .9. Average Jitter Analysis for Random Topology 


\section{CONCLUSION}

We in this paper present analysis on performance of coexistence heterogeneous networks. In this paper, we propose a new power based scheme using inter \& intra cluster scheduling mechanism for the coexistence of multiple IEEE 802.15.4 LRWPAN and IEEE 802.11b WLAN.The performance metrics of IEEE 802.15.4 network such as bit error,throughput,average end-end delay and average jitter is analyzed when the nodes are static. The simulation results show that the proposed scheme is effective in performance improvement for coexistence network of IEEE 802.15.4 for random topology. In future the analysis can be extended with mobility model and the same proposed scheme can be implemented with Exata emulator.

\section{REFERENCES}

[1] M. Petrova, et al, "IEEE 802.15.4 Low Rate - Wireless Personal Area Network Coexistence Issues," Proc. IEEE WCNC'06, Las Vegas, USA

[2] I. Howitt and J. A. Gutierrez, "Low-Rate Wireless Personal Area Networks - Enabling Wireless Sensors with IEEE 802.15.4," Proc. IEEE WCNC'03, vol.3, pp. 1481-1486

[3] S. Shin, et al, "Packet error rate analysis of IEEE IEEE 802.15.4 under IEEE 802.11b interference," Proc. WWIC'05, pp. 279-288

[4] A. Sikora, "Coexistence of IEEE 802.15.4 (ZigBee) with IEEE 802.11 (WLAN), Bluetooth, and Microwave Ovens in 2.4 $\mathrm{GHz}$ ISM-Band," web document, http://www.baloerrach.de/stzedn/

[5] Ling-Jyh Chen, Tony Sun, Mario Gerla," Modeling Channel Conflict Probabilities between IEEE 802.15 based Wireless Personal Area Networks", Communications, 2006. ICC apos; 06. IEEE International Conference on Vol.1, Issue, June 2006 Page(s):343 - 348
[6] Soo Young Shin, Hong Seong Parky, Sunghyun Choi, Wook Hyun Kwon," Packet Error Rate Analysis of IEEE 802.15.4 under IEEE $802.11 \mathrm{~b}$ Interference", IEICE Transactions on Communications 2007

[7] Dae Gil Yoon, Soo Young Shin, Wook Hyun Kwon and Hong Seong Park," Packet Error Rate Analysis of IEEE 802.11b under IEEE 802.15.4 Interference" Vehicular Technology Conference, 2006. VTC 2006-Spring. IEEE 63rd Publication Date: 7-10 May 2006 Volume: 3, On page(s): 1186-1190

[8] G.M.Tamilselvan, Dr.A.Shanmugam, "Modeling Channel Conflict Probabilities and Interference analysis of Coexistent Heterogeneous Networks" International Journal of Computer science and Knowledge Engineering (IJCSKE), Vol 3.Jan-June 2009, on page(s): 113-118

[9]. G.M.Tamilselvan, Dr.A.Shanmugam, "Probability Analysis of Channel Collision between IEEE 802.15.4 and IEEE 802.11b using Qualnet Simulation for Various Topologies" International Journal of Computer Theory and Engineering(IJCTE), Vol. 1, No. 1, April 2009, On page(s):59-64

[10]. G.M.Tamilselvan, Dr.A.Shanmugam, "Effect of Inter Packet Delay in Performance Analysis of Coexistence Heterogeneous Wireless Packet Networks", International Journal of Network Security and Applications (IJNSA), Vol 1, No 2, July 2009, On Page(s):40-49. 